AperTO - Archivio Istituzionale Open Access dell'Università di Torino

\begin{abstract}
Chitosan-Shelled Nanobubbles Irreversibly Encapsulate Morpholino Conjugate Antisense Oligonucleotides and Are Ineffective for Phosphorodiamidate Morpholino-Mediated Gene Silencing of DUX4
\end{abstract}

This is a pre print version of the following article:

Original Citation:

Availability:

This version is available http://hdl.handle.net/2318/1772923

since 2021-02-14T17:46:11Z

Published version:

DOI:10.1089/nat.2020.0862

Terms of use:

Open Access

Anyone can freely access the full text of works made available as "Open Access". Works made available under a Creative Commons license can be used according to the terms and conditions of said license. Use of all other works requires consent of the right holder (author or publisher) if not exempted from copyright protection by the applicable law. 


\title{
Chitosan-shelled nanobubbles irreversibly encapsulate morpholino conjugate antisense oligonucleotides and are ineffective for PMO-mediated gene silencing of DUX4
}

Maria Sofia Falzarano ${ }^{1 \wedge}$, Monica Argenziano ${ }^{2 \wedge}$, Anne Charlotte Marsollier ${ }^{3}$, Virginie Mariot ${ }^{3}$, Davide Rossi $^{2}$, Rita Selvatici ${ }^{1}$, Julie Dumonceaux ${ }^{3}$, Roberta Cavalli ${ }^{2}$, Alessandra Ferlini ${ }^{1,4^{*}}$

1. Unit of Medical Genetics, University of Ferrara, Ferrara, Italy

2. Department of Scienza e Tecnologia del Farmaco, University of Torino, 10125 Torino, Italy

3. NIHR Biomedical Research Centre, University College London, Great Ormond Street Institute of Child Health and Great Ormond Street Hospital NHS Trust, London, UK;

4. The Dubowitz Neuromuscular Centre, UCL Street Institute of Child Health, London, WC1N 1EH, United Kingdom

$\wedge$ These authors contributed equally

* Corresponding Author: A.F. (fla@unife.it)

\begin{abstract}
Orphan drugs, including antisense oligonucleotides (AONs), siRNAs/miRNAs, Cas9 nuclease and recombinant genes, have recently been made available for rare diseases. However, the main bottleneck for these new therapies is delivery. Drugs/synthetic genes need to reach the affected tissues with minimal off-target effects and immune reactions. AON molecules are currently delivered as backboned naked compounds or via viral vectors. Nanocarriers are considered promising vehicles, able to improve drug distribution by organ targeting and limiting safety issues. We tested perfluoropentane (PFP)-based nanobubbles (NBS) as vehicles for loading phosphorodiamidate morpholino (PMO) AON to suppress DUX4 expression in a FSHD cell model. In vitro cell-free analysis demonstrated a good loading capacity of PMO into NBs, while experiments in cell cultures showed lack of therapeutic effect since expression of DUX4 and its targets remained unmodified. We conclude that these types of chitosan-shelled NBs do not release PMO-AON and are therefore not ideal for PMO AON-related therapies.
\end{abstract}

\section{INTRODUCTION}


Over the past several decades, new molecules mainly based on nucleic acids were investigated for the development of new orphan drugs for curing rare diseases (RDs) [1]. mRNA is one of the most interesting and promising biological target to be modulated using novel drugs, especially by splicing modulation using antisense oligoribonucleotides (RNA-based therapeutics) [2]. Encouraging preclinical data primed the development of new RNA-based molecules, some of these already approved by the Food and Drug Administration (FDA) and/or the European Medicine Agency (EMA). Among these, two new drugs for treating two rare and severe genetic diseases: Eteplirsen (Sarepta), a 30-mer phosphomorpholidate oligonucleotide for the treatment of Duchenne muscular dystrophy (DMD) [3], and Nusinersen (Spinraza), a 18-mer phosphorothioate 2'-O-methoxyethoxy oligonucleotide, for the treatment of spinal muscular atrophy (SMA) [4]. Both molecules are antisense oligonucleotides (AONs) and target pre-mRNA by altering the splicing machinery in 2 different manners: Eteplirsen induces the omission (skipping) of exon 51 from the DMD mature transcript [5], while Nusinersen enhances the inclusion of exon 7 in the SMN2 transcript [6].

Other approaches using AONs have been described, including i) prevention of 5 'cap formation, ii) induction of mRNA degradation, iii) inhibition of protein translation and iv) modulation of the 3' end mRNA processing $[7,8]$.

One of the most important difficulties encountered for the development of these AONs remains their poor cellular uptake [9]. Moreover, the presence of barriers (i.e. blood-brain barrier, vascular endothelial barrier) can further limit and prevent the $\operatorname{AON}$ biodistribution $[5,10]$. Indeed, it is estimated that only $<1 \%$ of RNA AONs reaches the biological target by systemic delivery [11].

In order to improve the uptake of RNA molecules in the desired tissues with low toxicity and reduced off-target effects, several chemical modifications have been developed [4, 12]. During the last few years, the progress of nanotechnology has also made available different nanoparticles to increase the efficacy of drugs by improving the drug bioavailability. Depending on the material used, nanoparticles can be classified in different categories [13] and the most common types used for drug delivery are liposomes and Solid Lipid Nanoparticles (SLNs) [14], Polymer nanoparticles (including micelles, nanocapsules, nanospheres, colloids, dendrimers, core-shells) [15], Lipid-based Nanoparticles (LNPs) [16] and Carbon-based nanomaterials (graphene, nanodiamonds, fullerenes, nanotubes) [17].

We previously described the use of biocompatible polymer core-shell nanoparticles (NPs) to carry AONs for exon skipping application in DMD disease. We showed the capability of delivering 2'OMePS M23D AON in $m d x$ mice. In particular, intraperitoneal and even oral administrations of 
NP-AON complexes induced dystrophin restoration in mice. Nevertheless, we also showed that these NPs were not biodegradable and tend to accumulate following repeated doses, causing liver damage and animal death [18-22, unpublished data].

In this study, we used nanobubbles (NBs), fully biodegradable and biocompatible nanocarriers (patent WO 2015/028901 A1) [23]. NBs are spherical core/shell structures filled by gases or vaporizable compounds, such as perfluorocarbons, with sizes in the nanometer order of magnitude. Their structure consists of a core that is stabilized by a lipid, polymer or by an albumin shell. NBs have shown promising results as novel nanocarriers, with improved stability and high drug-loading capacity, as well as extravasation capability. Indeed, their small size allows the possibility of extravasation from the blood vessels into the surrounding tissues [24]. NBs can be loaded using genetic material following different methods $[25,26]$.

In this report, two nanoformulations of polymer-shelled NBs were prepared and PMO loading was investigated.

Facioscapulohumeral muscular dystrophy (FSHD), a rare genetic, autosomal dominant, muscular dystrophy [27], was adopted as the disease model. FSHD is a gain-of-function disease due to the aberrant expression of DUX4 (Double homeobox 4), a transcription factor that is not normally expressed in muscles [28]. DUX4 expression leads to cell toxicity and to the deregulation of several genes, including MBD3L2, ZSCAN4, DEFB103 and TRIM43 [27, 29].

An emerging therapeutic strategy aims at silencing the DUX4 gene to reduce its expression and consequently its toxicity in FSHD muscle cells. This approach acts via AON interference at the 3' end of DUX4 mRNA [7, 30, 31]. PMO oligomers CS3 (PMO-CS3) showed the best efficiency and lead to a significant decrease of DUX4 mRNA levels [7].

We tested the PMO-loaded NBs to assess whether this nanoformulation might be able to deliver the AON and improve the DUX4 silencing in in vitro experiments.

We selected PMO-CS3 loaded within NBs using two different strategies and transfected FSHD myogenic cells. The two tested nanoformulations exhibited a small particle size (about $300 \mathrm{~nm}$ ), very high PMO-CS3 encapsulation efficiency (96\%-99\%) and good stability in simulated cytosol and over storage. Nevertheless, when the efficacy of PMO-nanobubbles was evaluated in FSHD cells, no modification of DUX4 expression was observed, including the expression of genes downstream of DUX4 (TRIM43, MBD3L2 and ZSCAN4). We concluded that these types of chitosan-shelled NBs irreversibly bind PMO AON and were unable to release the AON molecule, therefore being poorly suitable for PMO AON-mediated gene silencing therapies. 


\section{MATERIAL AND METHODS}

\section{Materials}

All reagents were of analytical grade and obtained from Sigma-Aldrich (St. Louis, MO, USA) unless otherwise specified. Epikuron $200^{\circledR}$ was kindly provided by Cargill.

\section{$\underline{\text { Nanobubbles preparation }}$}

Chitosan-shelled NBs (NBs) were prepared tuning a method previously described, using perfluoropentane for the core [23]. Briefly, an ethanol solution containing Epikuron 200 and palmitic acid $(1 \% \mathrm{w} / \mathrm{v})$ was added to perfluoropentane and ultrapure water and then homogenized using an Ultra-Turrax ${ }^{\circledR}$ homogenizer (IKA, Konigswinter, Germany). Finally, a chitosan solution $(2.7 \% \mathrm{w} / \mathrm{v})$ at pH 5.0 was added dropwise under magnetic stirring. To obtain the PMO-CS3 loaded NBs, two loading strategies were considered. PMO molecules were located either at the interface between the core and the shell (Formulation A) or incorporated in the NB polymer shell (Formulation B). In Formulation A, a PMO-CS3 solution was added dropwise to the nanoemulsion and incubated under magnetic stirring (30 minutes in an ice bath). Then, the sample was coated with the chitosan layer. In Formulation B, the PMO-CS3 was incorporated within the chitosan shell of pre-formed NBs by incubation under magnetic stirring at $4^{\circ} \mathrm{C}$ for 30 minutes. Finally, a Pluronic F68 solution $(0.01 \% \mathrm{v} / \mathrm{v})$ was added to NB Formulation B as a stabilizing layer (Supplementary Fig. 1).

Different amounts of PMO were either incorporated at the interface between the core and the shell (Formulation A) or loaded after the coating with chitosan (Formulation B) as shown in Table 1.

\section{Loading capacity and encapsulation efficiency of PMO-CS3 in nanobubbles}

The amount of PMO-CS3 incorporated in the two NB formulations was determined spectrophotometrically at $260 \mathrm{~nm}$ using an UV-visible spectrophotometer (VICTOR X, Multilplate reader, Perkin Elmer, Waltham, MA, USA). The PMO concentration was calculated using the external standard method, referring to a PMO calibration curve.

A volume of PMO-loaded NBs was placed in an Amicon ${ }^{\circledR}$ Ultra-0.5 centrifugal filter device and centrifuged $\left(15000 \mathrm{rpm}, 20\right.$ minutes, $\left.4^{\circ} \mathrm{C}\right)$. The amount of free PMO in the filtrate was quantified spectrophotometrically. 
The encapsulation efficiency was calculated by subtracting the amount of free PMO from the initial amount added, according to the following equation:

Encapsulation efficiency $=(($ Total PMO-free PMO $)) /($ Total PMO $) \times 100$

To determine the loading capacity, a volume of PMO-loaded NBs underwent filtration using a centrifugal filter device (Amicon ${ }^{\oplus}$ Ultra-0.5) to separate NBs from aqueous medium. NBs were recovered and freeze-dried to obtain a dry powder. A weighted amount of freeze-dried PMO-loaded NB sample was dispersed in ultrapure acid water $(\mathrm{pH} 3.5, \mathrm{HCl} 0.1 \mathrm{M})$ and sonicated for 15 minutes to obtain the rupture of the NB structure. The sample was then centrifuged ( $15000 \mathrm{rpm}, 10 \mathrm{~min}$ ) and the supernatant was analyzed at the spectrophotometer $(260 \mathrm{~nm})$ to determine the PMO content in the NBs.

The loading capacity was calculated according to the equation:

Loading capacity $=($ PMO content in NBs) / (NB weight $) \times 100$

\section{Physico-chemical characteristics analysis of formulations}

Blank and PMO-loaded NB formulations were characterized in vitro to determine their physicochemical characteristics. The average diameter and polydispersity index of the samples were determined by dynamic light scattering, using a 90 Plus instrument (Brookhaven, NY). The analyses were carried out at a fixed angle of $90^{\circ}$ and a temperature of $25^{\circ} \mathrm{C}$. For the measurements, the samples were diluted with filtered water $(1: 30 \mathrm{v} / \mathrm{v})$. The zeta potential was measured by electrophoretic mobility using the same instrument. The diluted samples were placed in an electrophoretic cell where a $15 \mathrm{~V} / \mathrm{cm}$ electric field was applied. The morphology of NB formulations was evaluated by Transmission Electron Microscopy, using a Philips CM10 instrument (Philips, Eindhoven, the Netherlands). Samples were dropped onto a Formvar-coated copper grid and airdried prior to analysis.

\section{$\underline{\text { In vitro efficacy evaluation }}$}

The immortalized FSHD clone 54-12 has been characterized previously [32]. The cells were cultivated as described previously [31]. The differentiation into myotubes was induced by replacing the growing medium by DMEM supplemented with $10 \mu \mathrm{g} / \mathrm{ml}$ insulin. The transfections were performed 2 days after the induction of differentiation and the cells were harvested 2 days later. 
Transfections were performed using RNAiMAx or lipofectamine as previously described [31]. RNA extraction was performed using Trizol according to the manufacturer's protocol (Life Technologies). Reverse transcription and real-time PCR were performed as described previously [31, 33]. The sequences of the primers used for real-time PCR are: DUX4-F (GGCCCGGTGAGAGACTCCACA) and DUX4-R (CCAGGAGATGTAACTCTAATCCAGGTTTGC). B2M was chosen as a normalizer.

\section{$\underline{\text { In vitro physical stability of PMO-nanobubbles formulations }}$}

The physical stability of blank and PMO-loaded NBs was evaluated over time. The average diameter, Z-potential and morphology of the NB formulations stored at $-20^{\circ} \mathrm{C}$ were checked up to 1 year. In addition, the physical stability of the two types of NBs was also determined after their incubation in phosphate buffered saline (PBS) at $\mathrm{pH}$ 7.4.

Moreover, the in vitro stability of chitosan-shelled NBs was evaluated by gel retardation assay, using electrophoresis in an agarose gel. To mimic PMO, two siRNA-loaded NB formulations were incubated in simulated cytosol $(142 \mathrm{mM} \mathrm{KCl}, 5 \mathrm{mM} \mathrm{NaCl}, 5 \mathrm{mM} \mathrm{MgCl} 2,25 \mathrm{mM}$ Hepes-KOH $(\mathrm{pH}$ 7,2), $1 \mathrm{mg} / \mathrm{ml} \mathrm{BSA}$ ) at a $1: 1 \mathrm{v} / \mathrm{v}$ ratio over time $(24,72,96$ hours) [34]. At the fixed times, an aliquot of siRNA-loaded NBs was analyzed by gel retardation assay. The samples were loaded into agarose gel $(1 \% \mathrm{w} / \mathrm{v})$ stained with an ethidium bromide solution $(0.5 \mu \mathrm{g} / \mathrm{mL})$. The electrophoresis ran in TAE buffer ( $40 \mathrm{mM}$ Tris base, $20 \mathrm{mM}$ acetic acid and $1 \mathrm{mM}$ EDTA; $\mathrm{pH} 8.0$ ) at $60 \mathrm{~V}$ for one hour. A solution of free siRNA $(0.1 \mu \mathrm{g} / \mu \mathrm{L})$ was used as a positive control. The banding pattern was visualized using an ultraviolet transilluminator and photographed with a Polaroid camera.

\section{RESULTS AND DISCUSSION}

\section{Physico-chemical characteristics of NB formulations}

In this work, chitosan-shelled nanobubbles were designed for the delivery of PMO. The use of a nanocarrier has been proposed with the aim of incorporating nucleic acids, protecting them from degradation and favoring the cell uptake and the transfection efficiency $[35,36]$. The chitosan formulation is referred to as "nanobubbles" for sake of simplicity, but it would be more correct to use the term "nanodroplets" when the core is constituted of perfluoropentane, being a perfluorocarbon liquid at room temperature (boiling point $29^{\circ} \mathrm{C}$ ). 
The Table 2A reports the physical features of the NB formulations, either blank or PMO loaded. All chitosan-shelled NBs had an average diameter of about $300 \mathrm{~nm}$ and a positive surface charge (about +30 mV). No significant changes in the physico-chemical parameters were observed after PMO loading. After incubation in PBS at pH 7.4 the chitosan NBs showed a decrease of zeta potential value reaching $+10 \mathrm{mV}$, while no significant size change was determined. The surface charge of nanoparticles plays a crucial role in cell uptake. It is worth noting that this parameter is correlated with the amount of internalized nanocarriers. Interestingly, a number of studies focused on chitosan-based nanoparticles to optimize their biological interactions.

Yue et al. showed (Biomacromolecule) the strong correlation between the internalization and cellular trafficking of chitosan nanoparticles with different zeta potential values. The presence of a positive charge can promote the nanoparticle internalization rate, thanks to the interaction with the negative charge of the cellular membrane, enabling to overcome the biological barrier (Forest). Therefore, thetherapeutic effectiveness of positive charged nanosystems can be increased. This is very important especially for gene therapy being necessary the nucleic acid internalization to obtain an effect. The decrease of $65 \%$ of the surface charge of the NBs might decrease the contact with the cells, limiting their internalization.

TEM image showed a well-defined core-shell structure and spherical morphology of PMO-NBs (Supplementary Fig. 2). Both chitosan-shelled NBs were able to load PMO with similar encapsulation efficiency ranging from $96 \%$ up to $99 \%$ (Tab. 2B). The maximum encapsulation efficiency was achieved using the lowest amount of PMO-CS3 encapsulated in the polymer shell (Tab. 2B, c).

Loading capacity of $6.10 \%$ and $6.45 \%$ were obtained with NB formulation A and B, respectively. These results confirm the feasibility of preparing chitosan NBs for loading modified RNA (PMO) and support our previous results and other publications describing the use of NBs to incorporate DNA and small interfering RNAs (siRNA) [23, 37-39]. Taking into account the neutral charge of PMO backboned AON, non-electrostatic interactions such as hydrogen bonding and hydrophobic interactions are involved in the incorporation of PMO in NB structure.

We therefore further investigate the therapeutic effect of PMO-CS3 NBs nanoformulations on FSHD cell models to induce DUX4 gene silencing.

\section{$\underline{\text { In vitro therapeutic effect evaluation }}$}

In order to evaluate the NBs efficacy, $50 \mathrm{nM}$ of PMO-CS3 NBs was transfected in differentiated FSHD myotubes obtained from immortalized myoblasts. We first assessed two different formulations in 
which different amounts of PMO were either incorporated at the interface between the core and the shell or loaded after the coating with chitosan (Tab. 1).

The naked PMO-CS3 was used as a positive control. Naked PMO (Figure 1A, lines 6 and 7) causes an important decrease in DUX4 mRNA expression. Transcript reduction or modification was not observed with the PMO-CS3 NBs (Figure 1A, lines 8-12 and 14-18). The use of RNAiMaxReagant that is commonly used to transfect oligonucleotides did not improve the effect of the PMO-CS3 NBs (figure 1A w/o RNAiMax, lines 8-12; with RNAiMax, lines 14-18). We measured the expression of several genes downstream of DUX4 including TRIM43, MBD3L2 and ZSCAN4 [30]. The transfection using naked PMO-CS3 induced 25-85\% decreased expression of these genes (Fig. 1B). PMO-CS3 NBs were completely ineffective in the presence of RNAiMAx. However, PMO-CS3 NBs in the absence of RNAiMAx induce a decrease of TRIM43 (up to 25\%), MBD3L2 (29\%) and ZSCAN4 (56\%) (Fig. 1B). We next increased the NBs final concentration and performed a dose response for formulations (b) and (d). The impact of the presence of antibiotics in the differentiation medium was also assessed (Fig. 1, C and D) and transfections were done without RNAiMax. No modification of DUX4 mRNA expression was observed with NBs while using naked PMO-CS3 but a strong decrease was evident (Fig. 1C). The expression levels of the DUX4 network genes were not modified by PMO-CS3 NB (Fig. 1D). The presence/absence of antibiotics in the differentiation medium did not affect the transfection efficacy.

Both formulations were further modified (Tab. 1, $f$ and $g$ ) in order to load a similar PMO amount and $50 \mathrm{nM}$ of each optimized formulation were used to transfect FSHD myotubes. DUX4 expression was assessed by qPCR (Fig. 1E) and no reduction was observed compared to the control cells (transfected using NBs alone). Expression levels of the DUX4 network genes were unchanged (Fig. $1 F)$.

No toxicity was observed in FSHD myotubes as described in previous studies using the same NBs loaded with DNA molecules [23].

Based on these results, we can consider two hypotheses to explain the absence of cell transfection: I) The nanobubbles strongly encapsulate and retain the PMO avoiding its release and II) the nanobubble formulations are unable to be internalized in this type of cells due to their low surface charge. Since previous experiments showed the chitosan-shelled NB internalization capability in tumor cell lines (ref), we thus assessed the in vitro physical stability of PMO-CS3 NB formulations. 
The in vitro physical stability of NBs in simulated cytosol was evaluated by gel retardation assay performed on siRNA-loaded NBs to mimic the oligonucleotide incorporation within nanobubbles. The physical stability of the formulations was evaluated at different times: $24 \mathrm{~h}, 72 \mathrm{~h}$ and $96 \mathrm{~h}$. The electrophoretic mobility showed that both nanoformulations (NB A and NB B) fully retained the RNA molecules without releasing them over time in simulated cytosol (Supplementary Fig. 3). This behavior might suggest that both nanoformulations were stable and did not release the siRNA over time. We can speculate that same behavior also occurred for PMO molecules. This was observed both on the just-prepared nanoformulations as well as in those following storage at $-20^{\circ} \mathrm{C}$ for 1 year.

We demonstrated that NB loading experiments using PMO-CS3 were very promising because of the high loading capacity and the formulation stability, thus ensuring high protection to the AON. Nevertheless, the results of the in vitro stability experiment show that PMO is not released from the PMO-CS3 NBs from either the internal or external chitosan layers. Indeed, the "failure of releasing" hypothesis was supported by the complete lack of DUX4 gene silencing we observed using all NB formulations. We therefore conclude that PMO-CS3 NBs irreversibly load and do not make available any free, effective PMO AON to the cells.

A further possibility is that the very high amount of PMO AON loaded within the NBs, which is needed to achieve an encapsulation efficiency close to $100 \%$, together with the neutral charge of PMO backboned AON, may have a role in the "failure of releasing".

We may speculate that loading NBs with high quantity of PMO may change the interaction between NBs and AONs, and may induce the formation of extremely compact nanostructures that cannot release the PMO AON inside the cells.

Additionally, it would be necessary to evaluate the capability of PMO-loaded NBs to be internalized by myogenic cells. Future research will be focused on the NB shell optimization to promote their internalization.

Although we'll be able to design new NB formulations to evaluate whether a slow and sustained constant release of cargo is obtainable, our results robustly suggest that these types of chitosanshelled NBs are not appropriate in delivering PMO AON. Obviously, this result is disappointing, since PMO AON are extremely safe molecules and already used as approved orphan drugs. Many other AON types, negatively or positively charged (as for instance Pip-PMO [40]), might be good candidates for loading onto NBs. Alternatively, we may consider other NB shell compositions like synthetic polymers and albumin, which may modify this extremely high PMO affinity and consequent releasing failure. 


\section{REFERENCES}

1. Tambuyzer E, B Vandendriessche, CP Austin, PJ Brooks, K Larsson, K Miller Needleman, J Valentine, K Davies, SC Groft, R Preti, TI Oprea, M Prunotto. (2020). Therapies for rare diseases: therapeutic modalities, progress and challenges ahead. Nat Rev Drug Discov 19:93-111.

2. Bajan S, G Hutvagner. (2020). RNA-Based Therapeutics: From Antisense Oligonucleotides to miRNAs. Cells 9. pii: E137.

3. Aartsma-Rus A, AM Krieg. (2017). FDA approves Eteplirsen for Duchenne muscular dystrophy: the next chapter in the Eteplirsen Saga. Nucleic Acid Ther 27:1-3.

4. Martinovich KM, NC Shaw, A Kicic, A Schultz, S Fletcher, SD Wilton, SM Stick. (2018). The potential of antisense oligonucleotide therapies for inherited childhood lung diseases. Mol Cell Pediatr 5:3.

5. Echevarría L, P Aupy, A Goyenvalle. (2018). Exon-skipping advances for Duchenne muscular dystrophy. Hum Mol Genet 27:R163-R172.

6. Parente V, S Corti. (2018). Advances in spinal muscular atrophy therapeutics. Ther Adv Neurol Disord 11:1756285618754501.

7. Marsollier AC, R Joubert, V Mariot, J Dumonceaux. (2018). Targeting the Polyadenylation Signal of Pre-mRNA: A New Gene Silencing Approach for Facioscapulohumeral Dystrophy. Int J Mol Sci 19. pii: E1347.

8. DeVos SL, TM Miller. (2013). Antisense oligonucleotides: Treating neurodegeneration at the level of RNA. Neurotherapeutics 10:486-497.

9. Shen X, DR Corey. (2018) Chemistry, mechanism and clinical status of antisense oligonucleotides and duplex RNAs. Nucleic Acids Res 46:1584-1600.

10. Juliano RL. (2016). The delivery of therapeutic oligonucleotides. Nucleic Acids Res 44:6518-

48.

11. Godfrey C, LR Desviat, B Smedsrød, F Piétri-Rouxel, MA Denti, P Disterer, S Lorain, G NogalesGadea, V Sardone, R Anwar, S El Andaloussi, T Lehto, B Khoo, C Brolin, WM van Roon-Mom, A 
Goyenvalle, A Aartsma-Rus, V Arechavala-Gomeza. (2017). Delivery is key: lessons learnt from developing splice-switching antisense therapies. EMBO Mol Med 9:545-557.

12. Sardone V, H Zhou, F Muntoni, A Ferlini, MS Falzarano. (2017). Antisense OligonucleotideBased Therapy for Neuromuscular Disease. Molecules 22. pii: E563.

13. Falzarano MS, C Flesia, R Cavalli, C Guiot, A Ferlini. (2018). Nanodiagnostics and Nanodelivery Applications in Genetic Alterations. Curr Pharm Des 24:1717-1726.

14. Niu Z, I Conejos-Sánchez, BT Griffin, CM O'Driscoll, MJ Alonso. (2016). Lipid-based nanocarriers for oral peptide delivery. Adv Drug Deliv Rev 106:337-354.

15. Bennet D, Sanghyo K. Polymer Nanoparticles for Smart Drug Delivery. In: Application of Nanotechnology in Drug Delivery. Ali Demir Sezer, ed. (2014). Chapter 8, pp. 458-310.

16. Cheng X, RJ Lee. (2016). The role of helper lipids in lipid nanoparticles (LNPs) designed for oligonucleotide delivery. Adv Drug Deliv Rev 99:129-37.

17. Bhattacharya K, SP Mukherjee, A Gallud, SC Burkert, S Bistarelli, S Bellucci, M Bottini, A Star, B Fadeel. (2016). Biological interactions of carbon-based nanomaterials: From coronation to degradation. Nanomedicine 12:333-51.

18. Rimessi P, P Sabatelli, M Fabris, P Braghetta, E Bassi, P Spitali, G Vattemi, G Tomelleri, L Mari, D Perrone, A Medici, M Neri, M Bovolenta, E Martoni, NM Maraldi, F Gualandi, L Merlini, M Ballestri, L Tondelli, K Sparnacci, P Bonaldo, A Caputo, M Laus, A Ferlini. (2009). Cationic PMMA nanoparticles bind and deliver antisense oligoribonucleotides allowing restoration of dystrophin expression in the mdx mouse. Mol Ther 17:820-7.

19. Ferlini A, P Sabatelli, M Fabris, E Bassi, S Falzarano, G Vattemi, D Perrone, F Gualandi, NM Maraldi, L Merlini, K Sparnacci, M Laus, A Caputo, P Bonaldo, P Braghetta, P Rimessi. (2010). Dystrophin restoration in skeletal, heart and skin arrector pili smooth muscle of $\mathrm{mdx}$ mice by ZM2 NP-AON complexes. Gene Ther 17:432-8.

20. Bassi E, S Falzarano, M Fabris, F Gualandi, L Merlini, G Vattemi, D Perrone, E Marchesi, P Sabatelli, K Sparnacci, M Laus, P Bonaldo, P Rimessi, P Braghetta, A Ferlini. (2012). Persistent dystrophin protein restoration 90 days after a course of intraperitoneally administered naked 2'OMePS AON and ZM2 NP-AON complexes in mdx mice. J Biomed Biotechnol 2012:8970-76. 
21. Falzarano MS, C Passarelli, E Bassi, M Fabris, D Perrone, P Sabatelli, NM Maraldi, S Donà, R Selvatici, P Bonaldo, K Sparnacci, M Laus, P Braghetta, P Rimessi, A Ferlini. (2013). Biodistribution and molecular studies on orally administered nanoparticle-AON complexes encapsulated with alginate aiming at inducing dystrophin rescue in mdx mice. Biomed Res Int 2013:527418.

22. Falzarano MS, C Passarelli, A Ferlini. (2014). Nanoparticle delivery of antisense oligonucleotides and their application in the exon skipping strategy for Duchenne muscular dystrophy. Nucleic Acid Ther 24:87-100.

23. Cavalli R, A Bisazza, M Trotta, M Argenziano, A Civra, M Donalisio, D Lembo. (2012). New chitosan nanobubbles for ultrasound-mediated gene delivery: preparation and in vitro characterization. Int J Nanomedicine 7:3309-18.

25. Marano F, M Argenziano, R Frairia, A Adamini, O Bosco, L Rinella, N Fortunati, R Cavalli, MG Catalano. (2016). Doxorubicin-Loaded Nanobubbles Combined with Extracorporeal Shock Waves: Basis for a New Drug Delivery Tool in Anaplastic Thyroid Cancer. Thyroid 26:705-16.

26. Cavalli R, A Bisazza, D Lembo. (2013). Micro- and nanobubbles: a versatile non-viral platform for gene delivery. Int J Pharm Nov 456:437-45.

27. Chen JC, OD King, Y Zhang, NP Clayton, C Spencer, BM Wentworth, CP Jr Emerson, KR Wagner. (2016). Morpholino-mediated Knockdown of DUX4 Toward Facioscapulohumeral Muscular Dystrophy Therapeutics. Mol Ther 24:1405-11.

28. Das S, BP Chadwick. (2016). Influence of Repressive Histone and DNA Methylation upon D4Z4 Transcription in Non-Myogenic Cells. PLoS One 11:e0160022.

29. Yao Z, L Snider, J Balog, RJ Lemmers, SM Van Der Maarel, R Tawil, SJ Tapscott. (2014).

DUX4-induced gene expression is the major molecular signature in FSHD skeletal muscle. Hum Mol Genet 23: 5342-5352.

30. Ansseau E, C Vanderplanck, A Wauters, SQ Harper, F Coppée, A Belayew. (2017). Antisense Oligonucleotides Used to Target the DUX4 mRNA as Therapeutic Approaches in FaciosScapuloHumeral Muscular Dystrophy (FSHD). Genes (Basel) 8. pii: E93.

31. Marsollier AC, L Ciszewski, V Mariot, L Popplewell, T Voit, G Dickson, J Dumonceaux. (2016). Antisense targeting of $3^{\prime}$ end elements involved in DUX4 mRNA processing is an efficient 
therapeutic strategy for facioscapulohumeral dystrophy: a new gene-silencing approach. Hum Mol Genet. 25(8):1468-78.

32. Krom YD, J Dumonceaux, K Mamchaoui, B den Hamer, V Mariot, E Negroni, E Negroni, LN Geng, N Martin, R Tawil, SJ Tapscott, BG van Engelen, V Mouly, GS Butler-Browne, SM van der Maarel. (2012). Generation of isogenic D4Z4 contracted and noncontracted immortal muscle cell clones from a mosaic patient: a cellular model for FSHD. Am J Pathol 181:1387-1401.

33. Mariot V, R Joubert, C Hourde, L Feasson, M Hanna, F Muntoni, T Maisonobe, L Servais, C Bogni, R Le Panse, O Benvensite, T Stojkovic, PM Machado, T Voit, A Buj-Bello, J Dumonceaux. (2017). Downregulation of myostatin pathway in neuromuscular diseases may explain challenges of anti-myostatin therapeutic approaches. Nat Commun 8: 1859.

34. Lirussi D, Matrajt M. (2011). RNA Granules Present Only in Extracellular Toxoplasma Gondii Increase Parasite Viability. Int J Biol Sci 7: 960-967.

35. Rai R, Alwani S, Badea I. (2019). Polymeric Nanoparticles in Gene Therapy: New Avenues of Design and Optimization for Delivery Applications. Polymers 11(4). pii: E745.

36. Raemdonck K., Martens TF, Braeckmans K, Demeester J, De Smedt SC. (2013). Polysaccharide-based nucleic acid nanoformulations. Advanced drug delivery reviews 65: 11231147.

37. Ferrara KW, MA Borden, H Zhang. (2009). Lipid-shelled vehicles: engineering for ultrasound molecular imaging and drug delivery. Acc Chem Res 42: 881-892.

38. NA Geis, HA Katus, R Bekeredjian. (2012). Microbubbles as a vehicle for gene and drug delivery: current clinical implications and future perspectives. Curr Pharm Des 18:2166-2183.

39. Wu B, H Shang, X Liang, Y Sun, H Jing, X Han, W Cheng. (2019). Preparation of novel targeting nanobubbles conjugated with small interfering RNA for concurrent molecular imaging and gene therapy in vivo. FASEB J 33:14129-14136.

40. Betts C, AF Saleh, AA Arzumanov, SM Hammond, C Godfrey, T Coursindel, MJ Gait, MJ Wood. (2012). Pip6-PMO, A New Generation of Peptide-oligonucleotide Conjugates With Improved Cardiac Exon Skipping Activity for DMD Treatment. Mol Ther Nucleic Acids 1:e38. 


\section{ACKNOWLEDGMENTS}

MA, RC and DR thank University of Turin funding (ex 60\%) and COST Action (CA17103) for RC.

This study was financially supported by the FSHD society grant FSHS-22018-02. All research at Great Ormond Street Hospital NHS Foundation Trust and UCL Great Ormond Street Institute of Child Health is made possible by the NIHR Great Ormond Street Hospital Biomedical Research Centre. The views expressed are those of the author(s) and not necessarily those of the NHS, the NIHR or the Department of Health.

\section{AUTHOR DISCLOSURE STATEMENT}

None (J.D., A.C.M., V.M.)

None (M.S.F., R.S.)

None (M.A., R.C. and D.R.)

A.F. (Principal Investigator of Sarepta Clinical Trials)

\section{AUTHOR CONTRIBUTION}

M.S.F, A.F. and R.C designed the study

M.S.F., M.A., J.D, R.C., and A.F. wrote the paper

M.S.F. and M.A. conducted the preparation and characterization of nanoformulations

A.C.M, J.D and V.M. performed the in vitro efficiency studies

D.R. conducted the in vitro stability assay

M.S.F., M.A., J.D, A.F., R.S. and R.C analyzed the data

A.F. and R.C supervised the study 